\title{
Redundant Calibration: breaking the constraints of limited sky information
}

\author{
Ronniy C. Joseph ${ }^{1,2,3}$ \\ ${ }^{1}$ International Centre for Radio Astronomy Research, Curtin University, \\ Bentley, WA 6102, Australia. email: ronniy.joseph@icrar.org \\ ${ }^{2}$ ARC Centre of Excellence for All-sky Astrophysics (CAASTRO), Redfern, NSW, Australia \\ ${ }^{3}$ ARC Centre of Excellence for All Sky Astrophysics in 3D (ASTRO 3D), Perth, WA 6845
}

\begin{abstract}
The latest generation of low frequency radio interferometers, e.g. LOFAR, MWA, PAPER, has been pushing down the detection limits on the hydrogen signal from the Epoch of Reionisation. However, due to the challenges posed by foregrounds and instrumental systematics the signal has eluded detection thus far. To overcome these challenges we require a detailed understanding of the calibration of these relatively new telescopes. This led to a renewed interest in redundant calibration. Classical calibration schemes depend on sky models based on limited knowledge of the low frequency sky. Redundant calibration, however, allows us to escape our ignorance as it is sky model independent. We will review the field of redundant calibration, and present work we have undertaken to understand the limitations of this calibration method.
\end{abstract}

Keywords. instrumentation: interferometers, methods: numerical.

\section{Introduction}

The detection of the $21 \mathrm{~cm}$ signal from the Epoch of Reionisation (EoR) is a challenging experiment; we have to deal with large radiometric noise, bright foregrounds, and complex signal chains. The noise and the foregrounds hide the signal, and the signal chain leaves an instrumental imprint on the radio signals we are trying to measure. Only when we fully understand the impact of the instrument, we can mitigate it. We can then proceed to average over larger sets of data, building up to the required 1000 hours of observations, to achieve the desired signal to noise ratio. Accurate calibration of each observation is therefore a key element in the EoR experiment, but also in general to achieve the full potential of these new telescopes. Calibration solves for the unknown complex antenna gains $g_{i}$ describing the antenna imprints on the signal. Currently, we use the measured signal correlations $c_{i j}$ between antennas $i$ and $j$ forming a baseline pair, and models of the true correlations or visibilities $v_{i j}$ to solve for the antenna gains. These are related to each other through

$$
c_{i j}=g_{i}^{*} g_{j} v_{i j}+n_{i j},
$$

where the asterisk denotes complex conjugation and $n_{i j}$ is complex Gaussian noise.

These model based calibration methods rely on the completeness of our sky knowledge, which is lacking in this low frequency regime. Missing sources in source model catalogues impact our calibration solutions (Wijnholds et al. 2016), and these effects are enhanced in redundant arrays (e.g. PAPER, MWA, HERA), which contain many of the same baselines (Grobler et al. 2014). However, these redundant arrays do have extra sensitivity on scales probed by the redundant baselines. This makes them excellent for power spectrum measurements. This need for redundancy in the detection of the $21 \mathrm{~cm}$ power spectrum led to a resurgence of redundant calibration. As part of the MWA EoR experiment we are currently also undertaking research to study the applicability of redundant calibration. 


\section{Redundant Calibration Framework}

Redundant calibration relies on the presence of redundant baselines, i.e. pairs of antennas that measure the same visibility. Because a group of redundant baselines sample the same visibility $v$, the number of unknowns, e.g. gains $g$ and visibilities $v$, are lower in redundant arrays compared to non-redundant arrays. In theory, a sufficient amount of redundant baselines allows for a model-independent calibration of the antennas in the array. The concept is described in detail in Wieringa (1991) and has been further developed in Liu et al. (2010), Noorishad et al. (2012), Marthi \& Chengalur (2013), Zheng et al. (2014).

Currently, two linear implementations of redundant calibration, logcal and lincal, have gathered most of the attention within the community due their simplicity. They form the backbone of Omnical, which was developed as part of the MIT Epoch of Reionization experiment (MITEoR) (Zheng et al. 2014). It has been used to calibrate PAPER (Parsons et al. 2010) leading to their limits on the $21 \mathrm{~cm}$ signal (Ali et al. 2015). We are currently investigating the applicability of redundant calibration in the MWA (Tingay et al. 2013) and similar work is done for HERA (Dillon et al. 2016). Non-linear calibration has also been explored in (Marthi et al. 2013). Using steepest descent they find that non-linear least squares (NLS) can be faster than the linear methods, and that NLS produces noise unbiased results, similar to lincal. We will discuss noise bias in Section 3.2.

In logcal (Wieringa 1992) we take the logarithm of the complex quantities in Equation 1.1. This creates a system of equations in which the logarithm of the amplitudes and phases of each quantity, e.g. $\ln \left|g_{i}\right|$ and $\arg \left|g_{j}\right|$ respectively, are separated. Equation 2.1 shows the resulting two equations for the amplitudes and phases, when we ignore the noise term $n_{i j}$.

$$
\begin{aligned}
\ln \left|c_{i j}\right| & =\ln \left|g_{i}\right|+\ln \left|g_{j}\right|+\ln \left|v_{i j}\right| \\
\arg \left|c_{i j}\right| & =\arg \left|g_{j}\right|-\arg \left|g_{i}\right|+\arg \left|v_{i j}\right|
\end{aligned}
$$

Both systems of equations can be written in matrix form. We then solve the system using the general least square solution for a matrix equation of the form $\mathbf{A x}=\mathbf{b}$. The solution is given by $\hat{\mathbf{x}}=\left[\mathbf{A}^{T} \mathbf{A}\right]^{-1} \mathbf{A}^{T} \mathbf{b}$.

In lincal (Liu et al. 2010) we Taylor expand Equation 1.1 around an initial guess for the gains $g_{i}^{0}$ and $v_{i j}^{0}$, leading to

$$
c_{i j}=g_{i}^{0} g_{j}^{0 *} v_{i j}^{0}+g_{j}^{0 *} v_{i j}^{0} \Delta g_{i}+g_{i}^{0} v_{i j}^{0} \Delta g_{j}+g_{i}^{0} g_{j}^{0 *} \Delta v_{i j},
$$

where $\Delta g_{i}$ and $\Delta v_{i j}$ are the required corrections to our guesses. Analogous to the system of equations for logcal, we can write a similar matrix for the linearized framework, this time containing the real and imaginary components of our initial guesses $g_{i}^{0}$ and $v_{i j}^{0}$. But now we solve for $\Delta g_{i}$ and $\Delta v_{i j}$ to correct our guesses. This matrix has to be updated and inverted for each iteration, making this method quite computationally expensive.

\section{Implementation issues}

Omnical and software under development for HERA combine both linear implementations. They use logcal to get an initial guess and using lincal to further improve the solutions (Zheng et al. 2014). However, this has not completely resolved the issues associated with redundant calibration. In this section we will highlight some of these issues, and show examples which we encountered while investigating the applicability to the MWA. 

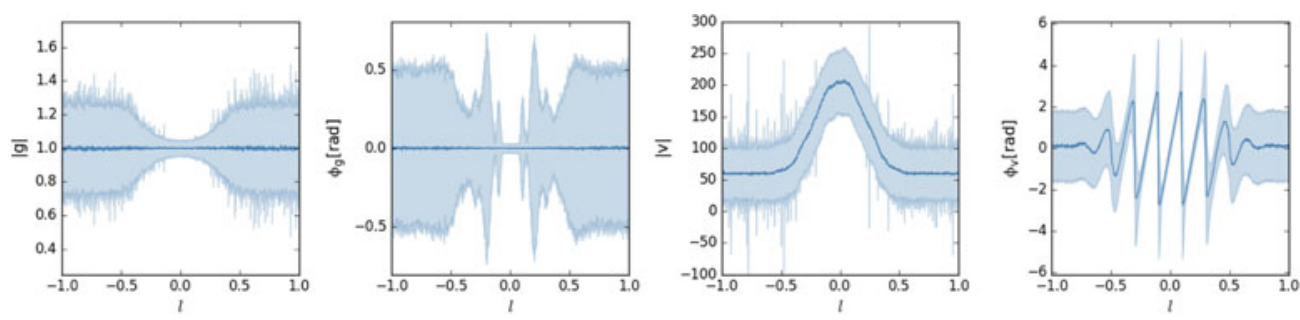

Figure 1. The logcal solutions for a simplistic 5 element interferometer with Gaussian-beams calibrated on a sky containing a $200 J y$ source and randomized background sky drawn from a source count distribution. From left to right: the amplitude gain $|g|$, the phase gain $\phi_{g}$ of a single antenna, the visibility amplitude $|v|$ and the visibility phase $\phi_{v}$ for a redundant visibility as a function of source position $l$. We show the solution mean (dark blue), and the 1-sigma solution variance(shaded blue). The mean of the amplitude solutions equals the input gain $|g|=1$, the amplitude solution variance inversely follows the shape of the beam, i.e. the variance increases when the beam response decreases. The mean of the phase solutions fluctuates around $\phi=0$ and jumps along with the variance at so-called phase wrapping points, which are further explained in the text. The visibility amplitude mean follows the shape of the Gaussian beam, caused by the source population variance and outliers caused by the noise variance. Similarly we see the visibility phase becoming more defined as the strong source moves into the beam, and we see its phase wrapping.

\subsection{Absolute Calibration}

Redundant calibration only yields relative solutions for the antenna gains. We can multiply the antenna gain amplitudes with some arbitrary factor $\alpha$ and simultaneously divide the visibility amplitude by $\alpha$ while keeping the system internally consistent. Similarly we can add an overall phase to each antenna gains, which will cancel out due to complex conjugation. Additionally we can also add an overall phase gradient to our antenna gains, as long as we subtract this from our visibilities. This final degeneracy corresponds to a rotation of the sky, i.e. redundant calibration can not determine the pointing of our telescope. (Wieringa 1992, Liu et al. 2010). To break these degeneracies we need some form of external calibration.

\subsection{Noise Bias}

Because logcal relies on the logarithm of Equation 1.1 it is noise biased. The real and imaginary components of the complex noise term are Gaussian-distributed, however, this is not true for the amplitude and the phase. This will lead to a systematic bias in the logcal solutions, and is also the reason why lincal was developed. We can work around the noise by averaging data over time scales on which the gain does not change much, but sufficient enough to lower the noise. (Liu et al. 2010)

\subsection{Phase Wrapping}

Phase wrapping adds an additional degeneracy to our complex solutions. We can add and subtract multiples of $2 \pi$ to our gains $g_{i}$ and visibilities $v_{i j}$ without changing the measured correlation $c_{i j}$, see Equation 1.1. This manifests itself in different ways for each linear implementation. In logcal phase wrapping shows itself by causing a large solution variance when certain redundant baseline groups measure a visibility phase of $\pi$. In lincal this will manifest itself by converging to a false minimum. In Figure 1 we show the solution variance of logcal in simulations. We attempt to calibrate a 5 element linear array on a randomised point source sky drawn from a source count distribution and a strong point source of $200 \mathrm{Jy}$. We show the solution variance as a function of the location of that strong source on the sky and we see that the variance for the phase 
increases when the measured phase wraps around. The amplitude variance depends on the $\mathrm{S} / \mathrm{N}$ of the amplitude, which is higher when the source is at phase centre. We need to unwrap the phases to some degree, before we apply logcal. Omnical uses the median phase within a redundant group. (Zheng et al. 2014) the HERA software currently uses products of visibilities.

\subsection{Convergence}

We have already mentioned that lincal can converge to the wrong minimum due to phase wrapping. But another reason why lincal might converge to a wrong solution are bad fiducial guesses. Combining both the logarithmic and linearised implementation was proposed to circumvent this. However, the aforementioned problem of phase wrapping makes logcal unstable on certain sky configurations, which gives lincal bad initial guesses. On top of that logcal is noise biased, causing additional issues.

\subsection{Non-Redundancy}

Finally, all radio telescopes are inherently non-redundant to some degree. There will be positions errors, and the antenna response patterns vary from antenna to antenna. The impact of these non-redundancy is currently being researched by the HERA and MWA collaborations.

\section{Solutions and Outlook}

The EoR community has been undertaking work to understand redundant calibration and its limiting factors. Here we have summarised literature to date and shown some initial results of the impact of the structure of the sky signal. The next step will be the inclusion of position offsets, and understanding the effects of beam variations.

But the need for some external calibration has motivated us to look at ways to combine sky models and redundancy. Some initial work into such a hybrid method has been mentioned in Sievers (2017), and we see this as the way forward in the era of the Square Kilometre Array.

\section{References}

Ali, Z. S. et al. 2015, The Astrophysical Journal, IOP Publishings, 809, 61

Dillon, J. S., \& Parsons, A. R., 2016, The Astrophysical Journal, IOP Publishings, 826, 181

Grobler, T. L. et al. 2014, Monthly Notices of the Royal Astronomical Society, Oxford University Press, 439, 4030-4047

Liu, A. et al. 2010, Monthly Notices of the Royal Astronomical Society, Oxford University Press, 408, 1029-1050

Noorishad, P. et al. 2012, Astronomy \& Astrophysics, EDP Sciences, 545, A108

Marthi, V. R. \& Chengalur, J. 2013, Monthly Notices of the Royal Astronomical Society, Oxford University Press,437,524-531

Parsons, A. R. et al. 2010, The Astronomical Journal, IOP Publishings,139,1468-1480

Sievers, J. L. 2017, arXiv:1701.01860

Tingay, S. J. et al. 2013, Publications of the Astronomical Society of Australia, Cambridge University Press, 30

Wieringa, M. H. 1992, Experimental Astronomy, Springer Nature, 2, 203-225

Wijnholds, S. J. et al. 1992, Monthly Notices of the Royal Astronomical Society, Oxford University Press, 457, 2331-2354

Zheng, H. et al. 2014, Monthly Notices of the Royal Astronomical Society, Oxford University Press,445, 1084-1103 